06

\title{
Согласованные нагрузки сантиметрового и миллиметрового диапазонов длин волн на СВЧ фотонных кристаллах
}

\author{
() Д.А. Усанов, ${ }^{1}$ В.П. Мещанов, ${ }^{2}$ А.В. Скрипаль, ${ }^{1}$ Н.Ф. Попова, ${ }^{2}$ Д.В. Пономарев, ${ }^{1}$ М.К. Мерданов ${ }^{3}$ \\ ${ }^{1}$ Саратовский национальный исследовательский государственный университет им. Н.Г. Чернышевского, \\ 410012 Саратов, Россия \\ ${ }^{2}$ Общество с ограниченной ответственностью Научно-производственное предприятие „НИКА-СВЧ“, \\ 410002 Саратов, Россия \\ ${ }^{3}$ Открытое акционерное общество Научно-производственный центр Электронное приборостроение, \\ 107014 Москва, Россия \\ e-mail: usanovda@info.sgu.ru
}

(Поступило в Редакцию 9 марта 2016 г.)

Созданы малогабаритные широкополосные согласованные нагрузки нового типа в виде разупорядоченных фотонных кристаллов, содержащих нанометровые металлические и диэлектрические слои. Для создания диэлектрических слоев с различными значениями диэлектрической проницаемости и малым значением тангенса угла диэлектрических потерь как в сантиметровом, так и в миллиметровом диапазонах длин волн предложено использовать композитные материалы, представляющие собой диэлектрические матрицы с наполнителем в виде воздушных включений.

DOI: $10.21883 /$ JTF.2017.02.44128.1794

\section{Введение}

Успешное развитие СВЧ-электроники во многом зависит от наличия соответствующей элементной базы. Одним из стандартных узлов современной аппаратуры СВЧ-диапазона, обеспечивающих, в частности, функционирование таких устройств, как направленные ответвители, сумматоры, измерители мощности, измерительные мосты, фильтры и т.д. [1,2], является согласованная (неотражающая) нагрузка. Наилучшую перспективу практического применения имеют нагрузки, которые обладают совокупностью таких свойств, как широкополостность, низкий уровень отражаемой и высокий рассеиваемой мощности, малые габариты. В качестве поглощающих элементов в согласованных нагрузках обычно используют ферроэпоксидные композиты, обладающие большими потерями, или диэлектрические стержни с нанесенными на них слоями резистивного материала. Существующие нагрузки не всегда удовлетворяют потребностям практики по габаритам, частотным характеристикам. Так, например, для обеспечения широкополосного согласования длина волноводных нагрузок должна в несколько раз превышать длину волны электромагнитного излучения во всем частотном диапазоне, в котором они используются, а геометрические размеры и электрофизические характеристики поглощающих элементов должны контролироваться с высокой точностью.

В сантиметровом диапазоне длин волн одним из недостатков существующих согласованных нагрузок являются их большие габариты, которые превышают в трехсантиметровом диапазоне длин волн $100 \mathrm{~mm}$. При переходе в миллиметровый диапазон длин волн при создании нагрузок возникают трудности в обеспечении широкополосности согласования, связанные с частотной зависимостью диэлектрической и магнитной проница- емостей ферроэпоксидных композитов и резистивных материалов, используемых в качестве поглощающих элементов.

Ряд новых возможностей при создании СВЧ согласованных нагрузок сантиметрового и миллиметрового диапазонов открывается при использовании свойств так называемых структур с фотонной „запрещенной“ (СВЧ фотонных кристаллов) [3,4]. Отметим, что наряду с зоной, запрещенной для распространения волн в определенном диапазоне частот, как правило вследствие почти полного отражения электромагнитной волны, для таких структур характерно наличие и „разрешенной“ зоны, в частотном диапазоне которой волна не испытывает отражения и распространяется с малыми потерями. Однако в случае присутствия в таких структурах проводящих слоев, например в виде нанометровых металлических пленок, при распространении волны в так называемой „разрешенной“ зоне происходит ее поглощение. Такое свойство фотонных кристаллов авторами [5-8] было использовано для создания малогабаритных (с продольными размерами, не превышающими одной длины волны) согласованных нагрузок, работающих в диапазонах частот 8.4-11.2 GHz (сечение волновода $23 \times 10 \mathrm{~mm}$ ) и $31.5-36.0 \mathrm{GHz}$ (сечение волновода $7.2 \times 3.4 \mathrm{~mm}$ ) с коэффициентом стоячей волны по напряжению КСВН (VSWR), меньшим 1.20.

Однако эти диапазоны частот составляют лишь часть стандартных диапазонов, для работы в которых предназначены волноводы указанных поперечных сечений, а достигнутые значения КСВН не удовлетворяют в полной мере потребности разработчиков элементной базы СВЧ.

Поэтому актуальной остается задача расширения диапазона рабочих частот малогабаритных согласованных нагрузок на основе фотонных кристаллов до диапазонов, 
соответствующих стандартным сечениям волноводов в возможно большем числе используемых на практике СВЧ-диапазонов.

\section{Теоретическая модель согласованной нагрузки на основе фотонного кристалла}

Предложенные нагрузки на основе фотонных кристаллов представляют собой короткозамкнутые с одной стороны отрезки прямоугольного волновода с размещенными в них структурами из чередующихся слоев диэлектриков с различными значениями толщины и диэлектрической проницаемости и нанометровых слоев металла с различными значениями толщины и удельной электропроводности.

Требования, предъявляемые к параметрам слоев, могут быть сформулированы в результате расчетов коэффициента отражения электромагнитной волны при ее нормальном падении на слоистую металлодиэлектрическую структуру с использованием матрицы передачи волны между областями с различными значениями постоянной распространения электромагнитной волны $\gamma_{j}$ и $\gamma_{j+1}[9-12]$ :

$$
\begin{aligned}
& \mathbf{T}\left(z_{j, j+1}\right)= \\
& \left(\begin{array}{ll}
\frac{\gamma_{j+1}+\gamma_{j}}{2 \gamma_{j+1}} \exp \left(\left(\gamma_{j+1}-\gamma_{j}\right) z_{j, j+1}\right) & \frac{\gamma_{j+1}-\gamma_{j}}{2 \gamma_{j+1}} \exp \left(\left(\gamma_{j+1}+\gamma_{j}\right) z_{j, j+1}\right) \\
\frac{\gamma_{j+1}-\gamma_{j}}{2 \gamma_{j+1}} \exp \left(-\left(\gamma_{j+1}+\gamma_{j}\right) z_{j, j+1}\right) & \frac{\gamma_{j+1}+\gamma_{j}}{2 \gamma_{j+1}} \exp \left(-\left(\gamma_{j+1}-\gamma_{j}\right) z_{j, j+1}\right)
\end{array}\right)
\end{aligned}
$$

которая связывает коэффициенты $A_{j}, B_{j}$ и $A_{j+1}, B_{j+1}$, определяющие амплитуды падающих и отраженных волн по обе стороны от границы $z_{j, j+1}$, соотношением

$$
\left(\begin{array}{l}
A_{j+1} \\
B_{j+1}
\end{array}\right)=\mathbf{T}\left(z_{j, j+1}\right)\left(\begin{array}{l}
A_{j} \\
B_{j}
\end{array}\right) .
$$

Коэффициенты $A_{N+1}$ и $B_{0}$, определяющие амплитуды волны, прошедшей через многослойную структуру, и волны, отраженной от нее, связаны с коэффициентом $A_{0}$, определяющим амплитуду падающей волны, следующим соотношением:

$$
\left(\begin{array}{c}
A_{N+1} \\
0
\end{array}\right)=\mathbf{T}_{N}\left(\begin{array}{l}
A_{0} \\
B_{0}
\end{array}\right),
$$

где $\mathbf{T}_{N}=\left(\begin{array}{ll}\mathbf{T}_{N}[1,1] & \mathbf{T}_{N}[1,2] \\ \mathbf{T}_{N}[2,1] & \mathbf{T}_{N}[2,2]\end{array}\right)=\prod_{j=N}^{0} \mathbf{T}_{j,(j+1)}=\mathbf{T}\left(z_{N, N+1}\right) \times$ $\times \mathbf{T}\left(z_{N-1, N}\right) \ldots \mathbf{T}\left(z_{1,2}\right) \mathbf{T}\left(z_{0,1}\right) \quad-\quad$ матрица передачи слоистой структуры, состоящей из $N$-слоев.

Коэффициент отражения $R=B_{0} / A_{0}$ электромагнитной волны, взаимодействующей со слоистой структурой, определяется через элементы матрицы передачи $\mathbf{T}_{N} \mathrm{c}$ помощью соотношения

$$
R=-\frac{\mathbf{T}_{N}[2,1]}{\mathbf{T}_{N}[2,2]} .
$$

КСВН рассчитывается по известному соотношению

$$
\operatorname{VSWR}=\frac{1+|R|}{1-|R|} .
$$

Для описания распределения электрического поля $E(z)$ в одномерном фотонном кристалле может быть использовано волновое уравнение вида

$$
\frac{\partial^{2} E(z)}{\partial z^{2}}+\gamma(z) E(z)=0
$$

где $\gamma(z)$ - постоянная распространения электромагнитной волны.

Решение волнового уравнения внутри каждой из областей одномерного фотонного кристалла с постоянной распространения электромагнитной волны $\gamma_{j}$ может быть представлено в виде суперпозиции падающих и отраженных волн и рассчитано с использованием следующего соотношения:

$$
\begin{aligned}
E_{j} & =\left(T_{j}[1,1] A_{0}+T_{j}[1,2] B_{0}\right) \exp \left(\gamma_{j}\left(z_{j+1}-z_{j}\right)\right) \\
& +\left(T_{j}[2,1] A_{0}+T_{j}[2,2] B_{0}\right) \exp \left(-\gamma_{j}\left(z_{j+1}-z_{j}\right)\right),
\end{aligned}
$$

где $T_{j}[1,1], T_{j}[1,2], T_{j}[2,1], T_{j}[2,2]$ - элементы матрицы передачи слоистой структуры, состоящей из $j$-последовательно расположенных слоев.

Полученные соотношения были использованы для определения КСВН при взаимодействии электромагнитной волны в сантиметровом и миллиметровом диапазонах с нагрузками на основе СВЧ фотонных кристаллов, выполненных в виде многослойных металлодиэлектрических структур, полностью заполняющих поперечное сечение волновода, а также для расчета распределения напряженности электромагнитного поля вдоль структуры фотонного кристалла.

\section{Компьютерное моделирование характеристик согласованных нагрузок на основе СВЧ фотонных кристаллов}

Как следует из результатов расчета, выполненных на основе описанной выше модели, использование фотонных кристаллов с нанометровыми металлическими слоями позволяет создать согласованные нагрузки, обеспечивающие в диапазонах частот $8.15-12.05 \mathrm{GHz}$, $12.05-17.44 \mathrm{GHz}, 17.44-25.95 \mathrm{GHz}$ величину коэффициента стоячей волны по напряжению менее 1.10 при линейных размерах нагрузок менее 15, 10 и $9 \mathrm{~mm}$ coответственно, и в диапазонах частот $25.95-37.50 \mathrm{GHz}$, $37.50-53.57 \mathrm{GHz}$ величину КСВН менее 1.15 при линейных размерах, не превышающих 7 и $6 \mathrm{~mm}$ соответственно.

Однако, как показывают расчеты, для получения таких результатов необходимо создавать не только нанометровые металлические слои с определенным значением поверхностного сопротивления, но и использовать диэлектрические слои, обладающие строго определенными 
значениями диэлектрической проницаемости и толщины. При этом желательно применять диэлектрические материалы, обладающие как в сантиметровом, так и в миллиметровом диапазонах длин волн малым значением тангенса угла диэлектрических потерь. Такая возможность открывается, если использовать для создания диэлектрических слоев широко применяемые на СВЧ диэлектрические материалы, такие как тефлон и поликор (керамика $\mathrm{Al}_{2} \mathrm{O}_{3}$ ), в которых путем создания воздушных включений обеспечивается получение требуемых значений эффективной диэлектрической проницаемости с высокой степенью точности [13].

Слои исследуемых фотонных кристаллов, содержащие большое число воздушных включений, можно рассматривать как композитные материалы, представляющие собой диэлектрические матрицы на основе керамики с наполнителем в виде воздушных включений. Известно, что диэлектрические свойства композитных материалов могут быть охарактеризованы величиной эффективной диэлектрической проницаемости $\varepsilon_{\text {eff }}$, определяемой величинами диэлектрических проницаемостей матрицы $\varepsilon_{1}$, наполнителя $\varepsilon_{2}$ и их объемными долями.

Размеры отверстий, их количество и расположение определяли величину эффективной диэлектрической проницаемости $\varepsilon_{\text {eff }}$ слоя композитного материала. Для определения величины эффективной диэлектрической проницаемости $\varepsilon_{\text {eff }}$ композитного материала по частотным зависимостям коэффициента пропускания решалась обратная задача с использованием метода наименьших квадратов [13]. При реализации этого метода находится такое значение параметра $\varepsilon_{\text {eff }}$, при котором сумма $S\left(\varepsilon_{\text {eff }}\right)$ квадратов разностей коэффициентов пропускания $D$ композитного материала, созданного на основе диэлектрической матрицы с наполнителем в виде воздушных включений, и коэффициентов пропускания $D\left(\varepsilon_{\mathrm{eff}}, f, n\right)$ однородного слоя с эффективной диэлектрической проницаемостью $\varepsilon_{\text {eff }}$

$$
S\left(\varepsilon_{\mathrm{eff}}\right)=\sum_{n}\left(|D|^{2}-\left|D\left(\varepsilon_{\mathrm{eff}}, f_{n}\right)\right|^{2}\right)^{2}
$$

становится минимальной.

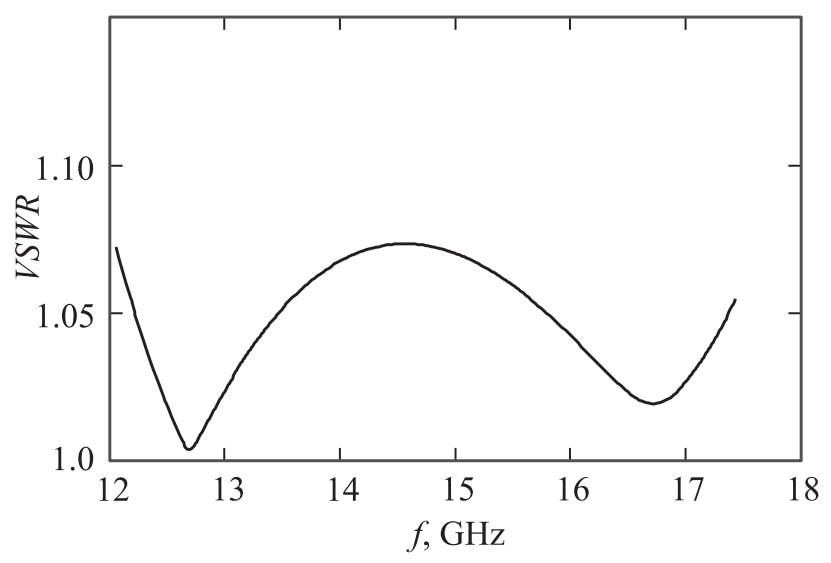

Рис. 1. Рассчитанная частотная зависимость КСВН в диапазоне $12.05-17.44 \mathrm{GHz}$.

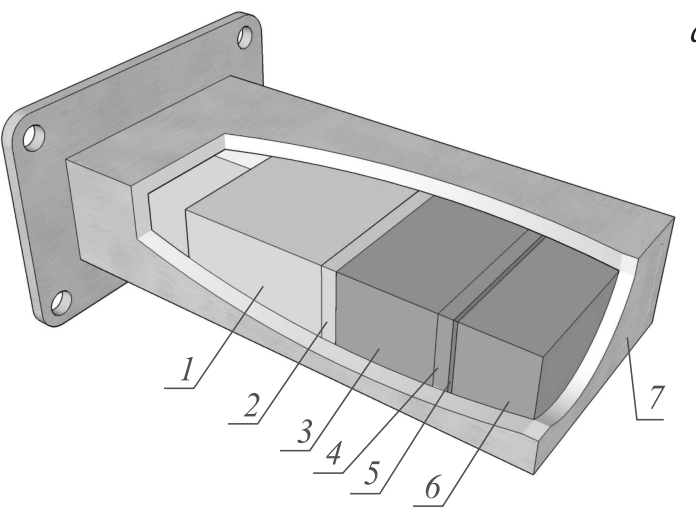

$a$

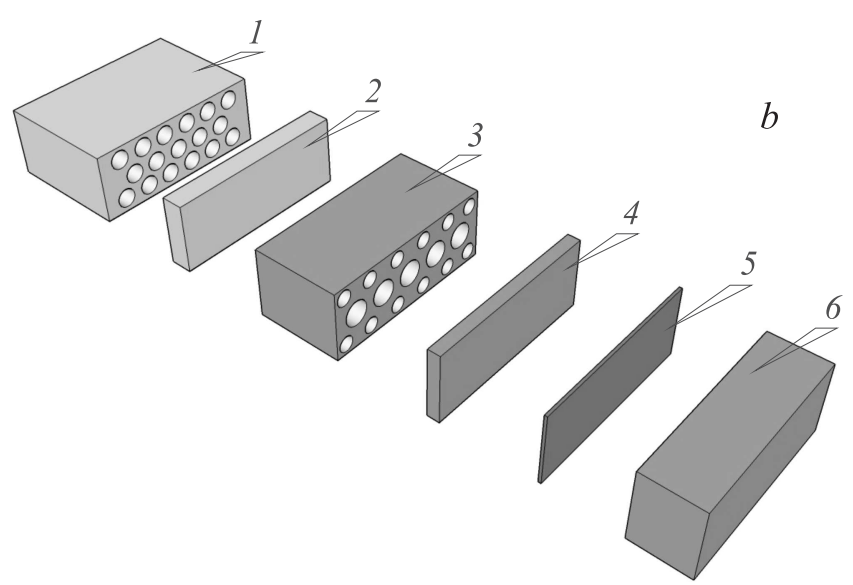

Рис. 2. Конструкция согласованной нагрузки (a) и металлодиэлектрической структуры $(b)$, включающей слои композитного материала $(\varepsilon: 1-1.4,3-6.15)$, сплошные диэлектрические слои $(\varepsilon: 2-2.0,4-9.6,6-9.6)$ и нанометровую металлическую пленку $-5(\rho=80 \Omega / \square)$.

Искомое значение эффективной диэлектрической проницаемости $\varepsilon_{\text {eff }}$ композитного материала определяется численным методом с помощью ЭВМ в результате решения уравнения

$$
\frac{\partial S\left(\varepsilon_{\mathrm{eff}}\right)}{\partial \varepsilon_{\mathrm{eff}}}=\frac{\partial\left(\sum_{n}\left(|D|^{2}-\left|D\left(\varepsilon_{\mathrm{eff}}, f_{n}\right)\right|^{2}\right)^{2}\right)}{\partial \varepsilon_{\mathrm{eff}}}=0 .
$$

Величина эффективной диэлектрической проницаемости композитного материала, созданного на основе диэлектрической матрицы с наполнителем в виде воздушных включений, может быть также рассчитана и с использованием ряда известных моделей „эффективной“ среды, описываемых соотношениями МаксвеллаГарнетта [14]

$$
\frac{\varepsilon_{\mathrm{eff}}-\varepsilon_{2}}{\varepsilon_{\mathrm{eff}}+2 \varepsilon_{2}}=x_{1} \frac{\varepsilon_{1}-\varepsilon_{2}}{\varepsilon_{1}+2 \varepsilon_{2}},
$$

Бруггемана [15]

$$
x_{1} \frac{\varepsilon_{1}-\varepsilon_{\mathrm{eff}}}{2 \varepsilon_{\mathrm{eff}}+\varepsilon_{1}}+x_{2} \frac{\varepsilon_{2}-\varepsilon_{\mathrm{eff}}}{2 \varepsilon_{\mathrm{eff}}+\varepsilon_{2}}=0,
$$




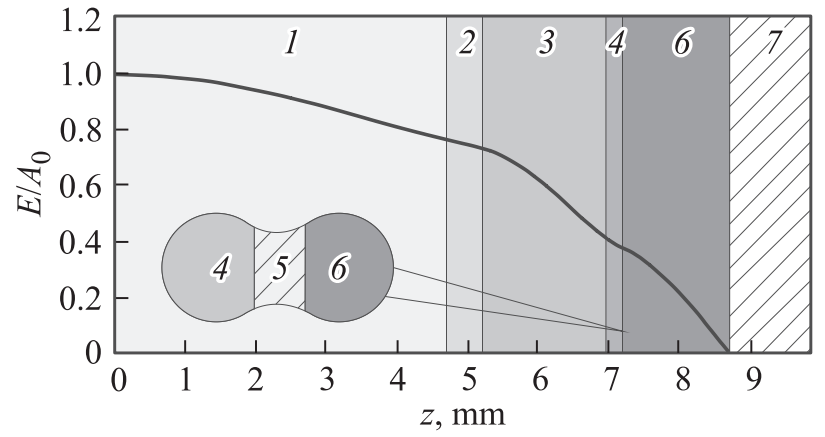

Рис. 3. Распределение напряженности электромагнитного поля на частоте $12.55 \mathrm{GHz}$ вдоль разупорядоченного фотонного кристалла. Слои композитного материала $(\varepsilon: 1-1.4,3-$ $6.15)$, сплошные диэлектрические слои $(\varepsilon: 2-2.0,4-$ 9.6, 6 - 9.6). Нанометровая металлическая пленка - 5 $(\rho=80 \Omega / \square)$.

Лихтенеккера [16]

$$
\log \varepsilon_{\mathrm{eff}}=\left(1-x_{1}\right) \log \varepsilon_{2}+x_{1} \log \varepsilon_{1} .
$$

Были выполнены расчеты в сантиметровых диапазонах $\quad 8.15-12.05 \mathrm{GHz}, \quad 12.05-17.44 \mathrm{GHz} \quad$ (рис. 1), $17.44-25.95 \mathrm{GHz}$ и миллиметровых диапазонах $25.95-37.50 \mathrm{GHz}, 37.50-53.57 \mathrm{GHz}$ КСВН согласованных нагрузок, выполненных в виде многослойных металлодиэлектрических структур (рис. 2), состоящих из чередующихся слоев композитного материала, созданного на основе диэлектрической матрицы с наполнителем в виде воздушных включений, характеризующегося эффективной диэлектрической проницаемостью, и нанометровой металлической пленки, нанесенной на керамическую подложку $\left(\mathrm{Al}_{2} \mathrm{O}_{3}\right)$.

Следует отметить, что предложенные согласованные нагрузки в виде фотонных кристаллов имеют разупорядоченную слоистую структуру, в которой диэлектрическая проницаемость слоев увеличивалась по направлению к короткозамыкающей стенке отрезка волновода.

Представляет интерес расчет распределения напряженности электромагнитного поля вдоль структуры фотонного кристалла, обеспечивающей величину коэффициента отражения, близкую к нулевому значению. Результаты расчета представлены на рис. 3 .

В разупорядоченной структуре при коэффициенте отражения, близком к нулевому значению, отсутствуют узлы и пучности в распределении напряженности электрического поля, и наблюдается монотонное уменьшение поля вдоль структуры, которое обращается в нуль в плоскости короткого замыкания.

\section{Результаты эксперимента}

По данным численного эксперимента были изготовлены широкополосные волноводные согласованные нагрузки на основе СВЧ фотонных кристаллов. Диэлектрические слои были выполнены из композитных материалов и представляли собой слои керамики $\left(\mathrm{Al}_{2} \mathrm{O}_{3}\right.$ с диэлектрической проницаемостью $\left.\varepsilon_{2}=9.6\right)$ и тефлона (с диэлектрической проницаемостью $\varepsilon_{2}=2.0$ ), в которых создавались массивы упорядоченных сквозных отверстий цилиндрической формы. Число отверстий было выбрано равным 17, при этом они были сгруппированы в шахматном порядке в три ряда, верхний и нижний по 6 отверстий, средний -5 отверстий. Нанометровые металлические слои $(\mathrm{Cr})$ напылялись на керамические подложки.

Измерения частотных зависимостей коэффициентов стоячей волны по напряжению (рис. 4) созданных согласованных нагрузок и предназначенных для использования в диапазонах частот $8.15-12.05 \mathrm{GHz}$ (рис. $4, a$ ), $12.05-17.44 \mathrm{GHz}$ (рис. $4, b), 17.44-25.95 \mathrm{GHz}$ (рис. $4, c)$ и $25.95-37.50 \mathrm{GHz}$ (рис. $4, d$ ) проводились с помощью
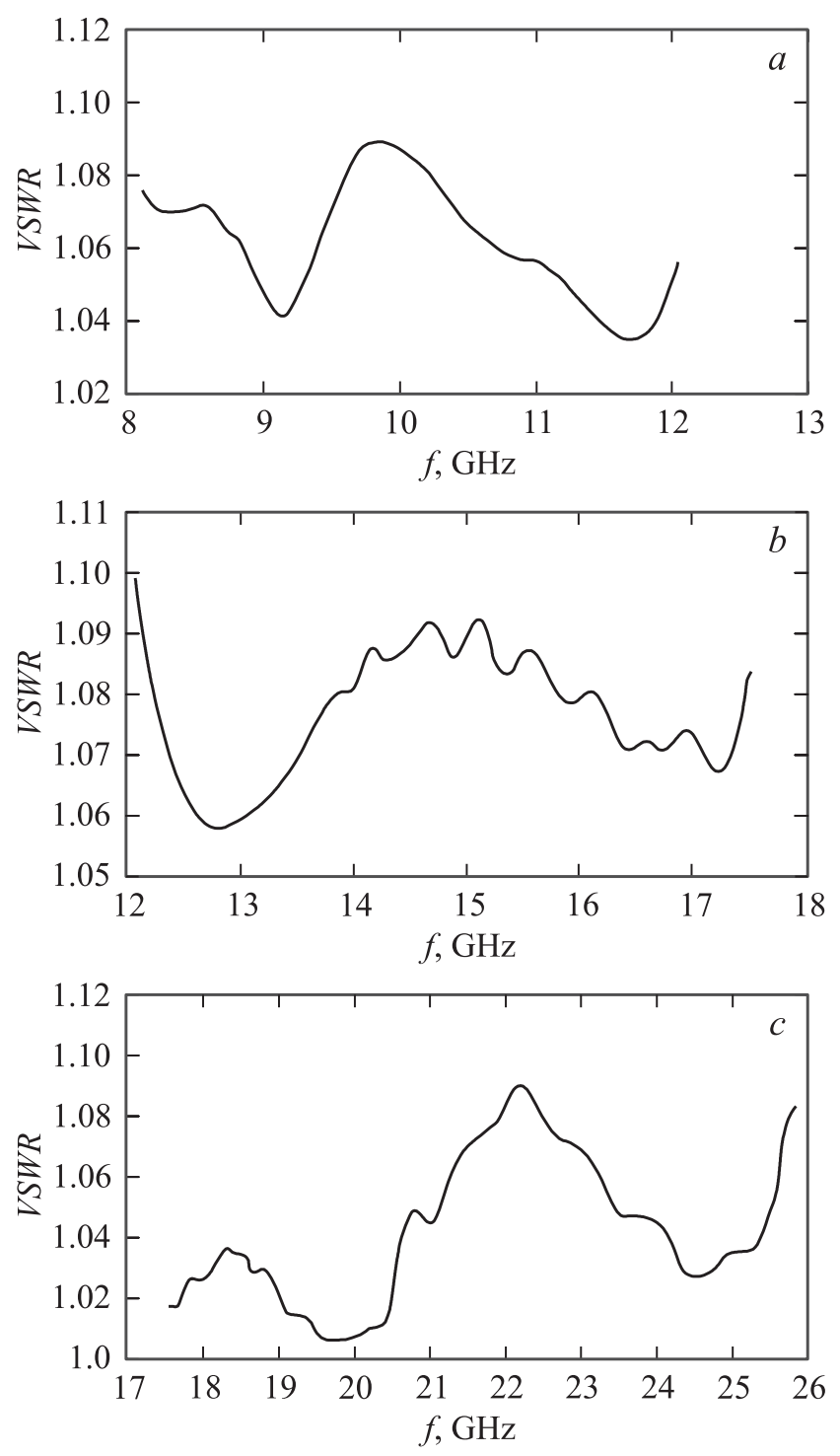

Рис. 4. $a-$ экспериментальная частотная зависимость КСВН в диапазоне $8.15-12.05 \mathrm{GHz} ; b-$ в диапазоне $12.05-17.44 \mathrm{GHz} ; c-$ в диапазоне $17.44-25.95 \mathrm{GHz} ; d-$ в диапазоне $25.95-37.50 \mathrm{GHz} ; e-$ в диапазоне $37.50-53.57 \mathrm{GHz}$. 

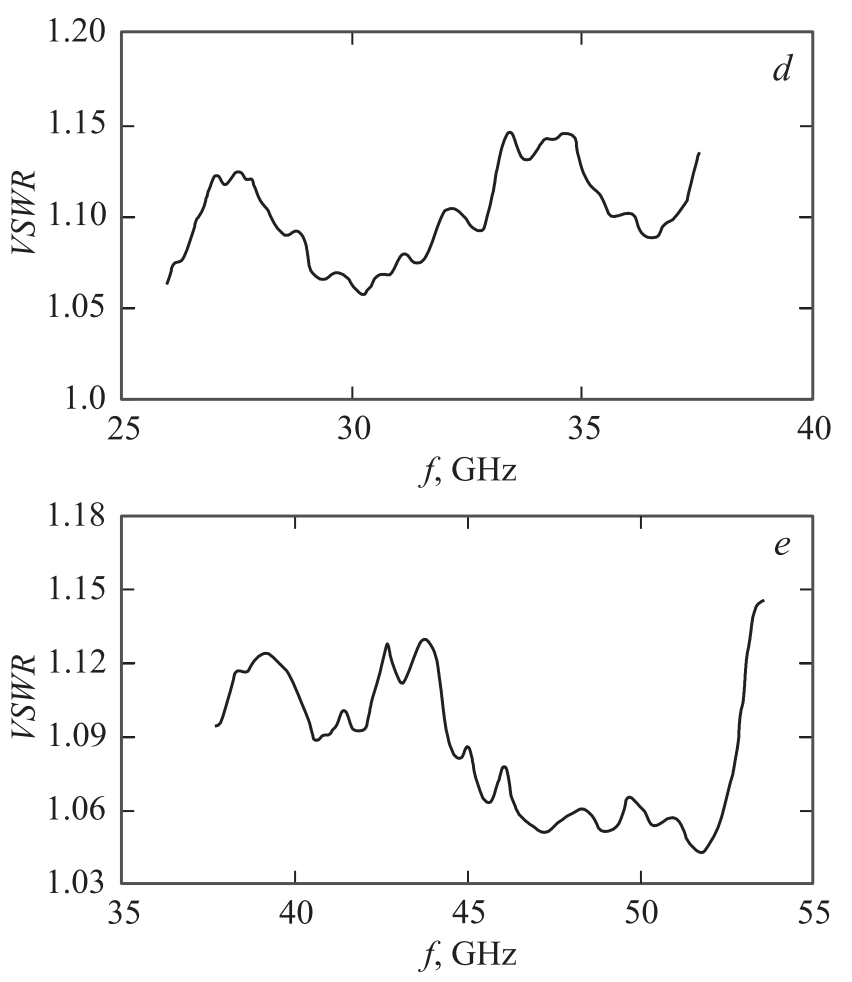

Рис. 4 (продолжение).

векторного анализатора цепей Agilent PNA N5230A и с помощью измерителя КСВН и ослабления Р2-68 в диапазоне $37.50-53.57 \mathrm{GHz}$ (рис. $4, e$ ).

Как следует из результатов эксперимента, созданные на основе СВЧ фотонных кристаллов согласованные нагрузки, предназначенные для использования в диапазонах $8.15-12.05 \mathrm{GHz}, 12.05-17.44 \mathrm{GHz}$, $17.44-25.95 \mathrm{GHz}$, имеют КСВН $<1.10$ при линейных размерах нагрузок менее 15,10 и $9 \mathrm{~mm}$ соответственно. Согласованные нагрузки для диапазонов частот $25.95-37.50 \mathrm{GHz}, \quad 37.50-53.57 \mathrm{GHz}$ имеют величину КСВН менее 1.15 при линейных размерах, не превышающих $7 \mathrm{~mm}$ и $6 \mathrm{~mm}$.

\section{Заключение}

Таким образом, показана теоретически и подтверждена экспериментально возможность создания в сантиметровом и миллиметровом диапазонах малогабаритных широкополосных согласованных нагрузок на основе СВЧ фотонных кристаллов, состоящих из чередующихся нанометровых металлических слоев с различными значениями толщины и электропроводности, и слоев композитных материалов, представляющих собой диэлектрические матрицы с наполнителем в виде воздушных включений.

Установлено, что для достижения значений КСВН согласованных нагрузок данного типа, удовлетворяющих требованиям, предъявляемым разработчиками элементной базы СВЧ, во всей полосе стандартных диапазо- нов частот, необходимо включать в состав слоистой структуры не только нанометровые металлические слои с определенным значением поверхностного сопротивления, но и использовать диэлектрические слои, обладающие строго определенными значениями диэлектрической проницаемости и толщины. Показано, что для создания диэлектрических слоев с различными значениями диэлектрической проницаемости и малым значением тангенса угла диэлектрических потерь как в сантиметровом, так и в миллиметровом диапазонах можно использовать композитные материалы, представляющие собой диэлектрические матрицы с наполнителем в виде воздушных включений.

Работа выполнена при финансовой поддержке Министерства образования и науки РФ (государственное задание № 1376 и 1575) и стипендии президента РФ (СП-2622.2015.3).

\section{Список литературы}

[1] Xelszajn J. Passive and Active Microwave Circuits. N.Y., Chichester, Brisbane, Toronto: John Wiley \& Sons, 1978. $284 \mathrm{p}$.

[2] Lee K.A., Guo Y., Stimson Ph.A., Potter K.A., Chiao JungChih, Rutledge D.B. // IEEE T. Anten. Propag. 1991. Vol. 39. N 3. P. 425-428.

[3] Joannopoulos I.D., Villenneuve Pierre R., Fan S. // Nature. 1997. Vol. 386. N 13. P. 143-149.

[4] Yablonovitch E., Gmitter T.J., Leung K.M. // Phys. Rev. Lett. 1991. Vol. 67. N 17. P. 2295-2298.

[5] Usanov D.A., Skripal A.V., Abramov A.V., Bogolubov A.S., Skvortsov V.S., Merdanov M.K. // Proc. of the XVII Intern. Conf. on Microwaves, Radar and Wireless Communications. Wroclaw, Poland. 2008. Vol. 2. P. 553-556.

[6] Usanov D.A., Skripal A.V., Abramov A.V., Bogolubov A.S., Skvortsov V.S., Merdanov M.K. // Proc. of the $38^{\text {th }}$ European Microwave Conf. Amsterdam. Netherland. 2008. P. 484-487.

[7] Усанов Д.А., Скрипаль А.В., Абрамов А.В., Боголюбов А.С., Сквориов В.С., Мерданов М.К. // Изв. вузов. Радиоэлектроника. 2009. № 1. С. 73-80.

[8] Пат. РФ № 2360336. Широкополосная волноводная согласованная нагрузка / Д.А. Усанов, А.В. Скрипаль, А.В. Абрамов, А.С. Боголюбов, В.С. Скворцов, М.К. Мерданов. 2009. БИ № 18.

[9] Усанов Д.А., Скрипаль А.В., Абрамов А.А., Боголюбов А.С. // ЖТФ. 2006. Т. 76. Вып. 5. С. 112-117.

[10] Чапльгин Ю.А., Усанов Д.А., Скрипаль А.В., Абрамов А.В., Боголюбов А.С. // Изв. вузов. Электроника. 2006. № 6. C. 27-35.

[11] Usanov D.A., Skripal Al.V., Abramov A.V., Bogolyubov A.S., Kalinina N.V. // Proc. of $36^{\text {rd }}$ European Microwave Conf. Manchester, UK, 2006. P. 921-924.

[12] Усанов Д.А., Скрипаль А.В., Абрамов А.В., Боголюбов А.С., Сквориов В.С., Мерданов М.К. // Изв. вузов. Электроника. 2007. № 6. С. 25-32.

[13] Усанов Д.А., Скрипаль А.В., Мерданов М.К., Горлищкий B.О. // ЖТФ. 2016. Т. 86. Вып. 2. С. 65-70.

[14] Maxwell-Garnett J.C. // Philos. T. Roy. Soc. Lond. A. 1904. Vol. 203. P. 385-420.

[15] Bruggeman D.A.G. // Annalen der Physik. (Leipzig) 1935. F. 5. B. 24. H. 8. S. $636-679$.

[16] Lichtenecker K. // Physikalische Zeitschrift. 1926. B. 27. H. 4. S. $115-158$. 